\title{
Magnetic resonance spectroscopy of atypical diffuse pontine masses
}

\author{
Mark D. Krieger, M.D., Stefan Blüml, Ph.D., And J. Gordon MCComb, M.D. \\ Division of Neurosurgery, and Department of Radiology, Childrens Hospital Los Angeles; and \\ Department of Neurological Surgery, Keck School of Medicine, University of Southern California, Los \\ Angeles, California
}

\begin{abstract}
Object. Diffuse pontine gliomas in children carry a dismal prognosis, with a mean survival of less than 1 year despite therapy. The diagnosis is based on the characteristic changes demonstrated on traditional magnetic resonance (MR) imaging. A few typically MR imaging-appearing pontine masses, however, do not behave in the expected fashion, which calls the original diagnosis into question.

Methods. The authors conducted a retrospective review of data obtained in 42 children (age 6 months-13 years) in whom diffuse pontine glioma had been diagnosed at their institution. Five of these patients (12\%) survived longer than expected ( $>18$ months). There were no differences in these patients in terms of demographics, presentation, traditional imaging findings, or treatment compared with the group as a whole. Magnetic resonance spectroscopy, however, demonstrated two distinct patterns not seen in typical diffuse pontine gliomas. In two patients elevated lipid and lactate levels were shown, with decreased levels of choline, myoinositol, and N-acetyl-aspartate (NAA). In the other patients strikingly elevated choline/creatinine ratios and myoinositol levels were observed in comparison with typical pontine tumors.

Conclusions. These MR spectroscopy patterns demonstrated in this retrospective study seem to convey prognostic information and may lead to an expansion of this diagnostic tool.
\end{abstract}

\section{KEY WORDS - magnetic resonance spectroscopy - diffuse pontine glioma • pediatric neurosurgery}

Brainstem gliomas comprise approximately 10 to $15 \%$ of childhood brain tumors. They typically present during early childhood, with a peak incidence between the ages of 5 and 8 years. The presentation is usually subacute, with progressive neurological impairment developing over days to weeks. Signs and symptoms are most commonly due to disruption of function of the brainstem and/ or cranial nerves.

In most cases, these are intrinsic tumors, arising within the neural substrate of the pons. The majority of tumors in the brainstem are high-grade gliomas that invade the brainstem, although low-grade gliomas also occur. ${ }^{3,14} \mathrm{~Pa}$ tients with diffuse tumors intrinsic to the pons have an extremely poor prognosis. With standard radiotherapy alone, the median time to progression is 5 to 6 months, and the median survival time is less than 1 year. The acuity of symptom onset is predictive of survival. ${ }^{5,6}$

Histological diagnosis is not undertaken in the majority of cases. ${ }^{1}$ Three major reasons exist for proceeding with treatment without first examining a biopsy sample. First, these are intrinsic lesions located in extremely eloquent neural tissue. Biopsy sampling would thus carry risks of inducing brainstem dysfunction. Second, the typical appearance of these lesions on MR images is considered

Abbreviations used in this paper: $\mathrm{CSF}=$ cerebrospinal fluid; $\mathrm{MR}=$ magnetic resonance; NAA $=\mathrm{N}$-acetyl-aspartate. diagnostic, and further workup has been proven inefficacious. Third, in intrinsic pontine gliomas of childhood, histological type has not been shown to affect outcome. ${ }^{19}$ Although the MR images are highly predictive of these tumors, other lesions with similar radiographic appearances may occur. In rare cases, inflammatory and infectious lesions, or other tumor types may develop, with important treatment and prognostic implications..$^{25}$

Although the true course of the disease is invariably fatal, 6 to $10 \%$ of children live more than 2 years after undergoing radiotherapy. ${ }^{10}$ Various theories have been proposed to account for this subset of patients. It is possible that these represent tumors with either less biological activity and are thus more slow growing, or are more sensitive to radiotherapy. Alternatively, these patients may harbor lesions other than tumors, although the appearance mimics that of tumors on MR imaging.

The goal of the present study was to evaluate ${ }^{1} \mathrm{H}-\mathrm{MR}$ spectroscopy as a means to gain additional information regarding the biological activity of diffuse pontine gliomas. Proton MR spectroscopy can be performed using standard MR imagers combined with a software upgrade; it adds approximately 10 minutes to imaging time. Unlike conventional MR imaging, which demonstrates protons predominantly in water, proton MR spectroscopy can quantify the relative concentration of nonwater, protoncontaining metabolites from discreet tissue regions by 
suppressing the signals from water and lipids. ${ }^{9,12,23}$ Various substances within the brain are measured using MR spectroscopy, including the neuronal marker NAA, creatine (a reflection of cellular metabolism), and choline (a constituent of cell membranes). Proton MR spectroscopy has been described in the evaluation of stroke, as well as neoplastic, infectious, and inflammatory diseases of the brain. $2,4,11,13,18,20,21,24$

Our hypothesis was that the chemical makeup of such tumors might reflect their biological activity and that MR spectroscopy could thus aid in the evaluation of these lesions.

\section{CLINICAL MATERIALS AND METHODS}

A retrospective review was conducted of the brain tumor database at Childrens Hospital Los Angeles of patients treated between January 1991 and January 2000. All cases of diffuse pontine glioma in this database were identified. Charts and imaging studies were reviewed in their entirety.

\section{Patient Population}

Forty-two pediatric cases of diffuse pontine glioma were identified. This group included 23 boys and 19 girls who ranged in age from 6 months to 13 years. All patients underwent MR imaging, which led to the diagnosis. Five of these patients $(12 \%)$ survived longer than expected $(>$ 18 months). There were no differences in these patients in terms of demographics, presentation, conventional imaging findings, or treatment compared with the group as a whole.

Informed parental consent was obtained to conduct an additional MR spectroscopy study after completion of all clinically indicated MR imaging studies. The MR imaging/MR spectroscopy protocol, and the review and correlation with clinical data were approved by the local institutional review board.

\section{Magnetic Resonance Spectroscopy Studies}

Precontrast and postcontrast MR imaging and ${ }^{1} \mathrm{H}-\mathrm{MR}$ spectroscopy images were acquired on a clinical 1.5-tesla General Electric imager in which a standard head coil was used. Single-voxel ${ }^{1} \mathrm{H}$-spectra of the tumors were acquired using a PRESS sequence (TE $35 \mathrm{msec}$, TR 1.5 seconds). Proton spectra were processed using the LC-Model software (Stephen Provencher Inc., Oakville, ON, Canada). The unsuppressed water was used as an internal reference and absolute intensities were obtained. The basis set consisted of 15 components: alanine, aspartate, choline, creatine, gamma-amino-butyrate, glucose, glutamine, glutamate, myoinositol, lactate, NAA, NAA glutamate, scylloinositol, taurine, and phosphorylethanolamine. Singlevoxel ${ }^{1} \mathrm{H}-\mathrm{MR}$ spectroscopy included the determination of the amounts of tissue and CSF (or fluid of necrotic regions within lesions). This measurement was based on the differences in the $\mathrm{T}_{2}$-weighted relaxation time of tissue water and CSF and has been explained in detail. ${ }^{7}$

Control data were obtained in seven individuals studied at this institution with unrelated and comparably minor clinical indications for MR imaging such as developmental delay or seizures activity and selected based on a "normal" MR imaging report.

\section{RESULTS}

We identified 42 children with diffuse pontine glioma ( 23 boys and 19 girls), who ranged in age from 6 months to 13 years (median age 4.5 years). The duration of symptoms, based on reports provided by their parents, ranged from 1 day to 7 months. In 37 of the 42 patients cranial nerve deficits were observed on initial examination. Long tract signs were present in 29 patients and ataxia in 31 patients.

Magnetic resonance images were obtained in all children prior to therapy. In all cases a diffuse tumor was seen centered in the pons. Cases involving isolated tectal lesions and dorsally exophytic lesions were excluded. All lesions appeared hyperintense on $\mathrm{T}_{2}$-weighted sequences. Ring or patchy enhancement was revealed on imaging prior to therapy.

In the entire group, the mean time from diagnosis to death was 9.5 months. Five (12\%) of these patients, however, survived longer than expected ( $>18$ months). There were no differences in patients in terms of demographics, presentation, traditional imaging findings, or treatment compared with the group as a whole. Three of these patients and 17 of the remaining 37 patients underwent CSF evaluation; in no case were the results suggestive of an alternate pathological processes.

High-quality ${ }^{1} \mathrm{H}-\mathrm{MR}$ spectroscopy were obtained in all five patients, as well as in 12 of the other 37 patients with typical gliomas. The overall evaluation session, including complete MR imaging and ${ }^{1} \mathrm{H}-\mathrm{MR}$ spectroscopy study times, required approximately 1 hour, and no adverse effects were reported.

A spectrum from healthy gray matter obtained in a control and that obtained in a diseased patient with a typical clinical course are shown in Fig. 1. In these tumors the choline level was increased relative to creatine whereas NAA was depleted. Myoinositol relative to creatine was increased (Fig. 1).

The spectral patterns demonstrated by ${ }^{1} \mathrm{H}-\mathrm{MR}$ spectroscopy in patients with atypically long survival proved to be different, which most likely represent significant variations in underlying biochemistry. A significantly elevated lipid peak, with a marked decrease in the other measured entities, was observed in two long-term survivors despite a typical MR imaging-based appearance of diffuse pontine glioma (Fig. 2).

In the other three long-term survivors, less dramatic shifts were revealed on MR spectroscopy. In these patients moderately elevated myoinositol and choline levels were displayed (Fig. 3).

\section{DISCUSSION}

Five $(12 \%)$ of the 42 patients with pontine gliomas at our institution lived longer than expected. This rate of atypical long-term survival is well described in the literature..$^{8,10,15}$ The reasons for these cases of long-term survival remain unknown, partly because of the inhomogeneity apparent in the literature. It is well documented that exophytic and tectal tumors have a significantly different disease course from true diffuse pontine gliomas, as do evenly enhancing pilocytic astrocytomas. ${ }^{8,17}$ Thus, their inclusion in some series would skew the results to indi- 

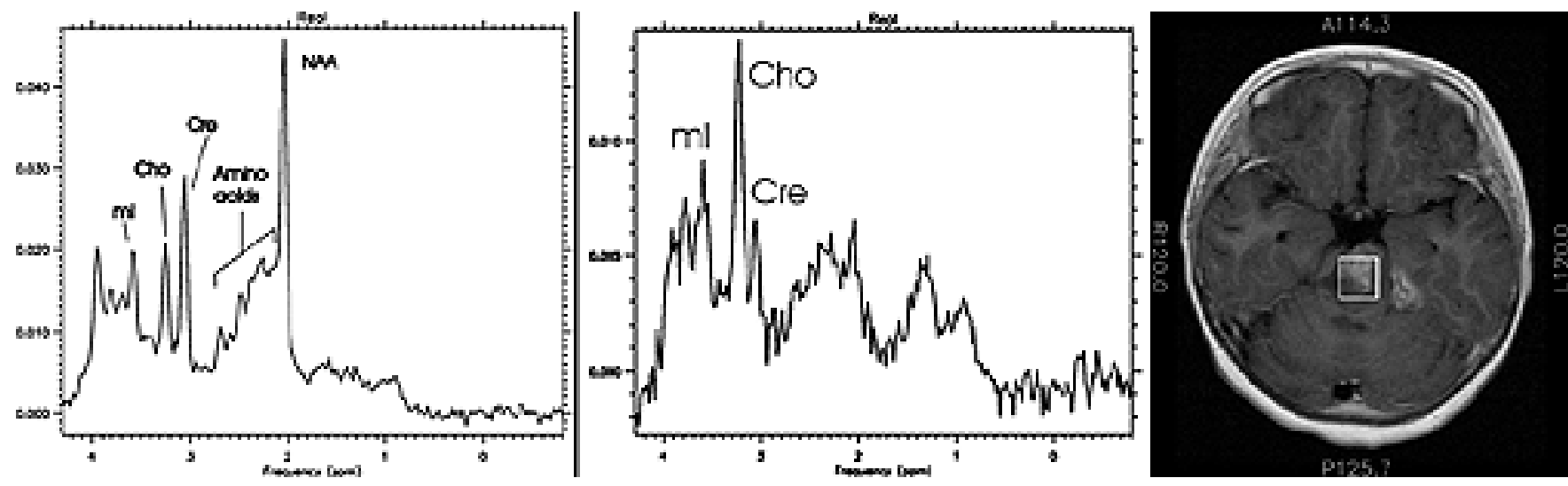

Fig. 1. Typical MR spectroscopy appearance for pontine glioma (right). Levels of choline, creatine, and myoinositol where elevated in comparision with spectra from observed in a "healthy" brain (left). Cho $=$ choline; $\mathrm{Cr}=\mathrm{creatine} ; \mathrm{mI}=$ myoinositol.

cate a greater number of long-term survivors. In our series, only patients with truly diffuse pontine gliomas on documented MR imaging were included to avoid this difficulty.

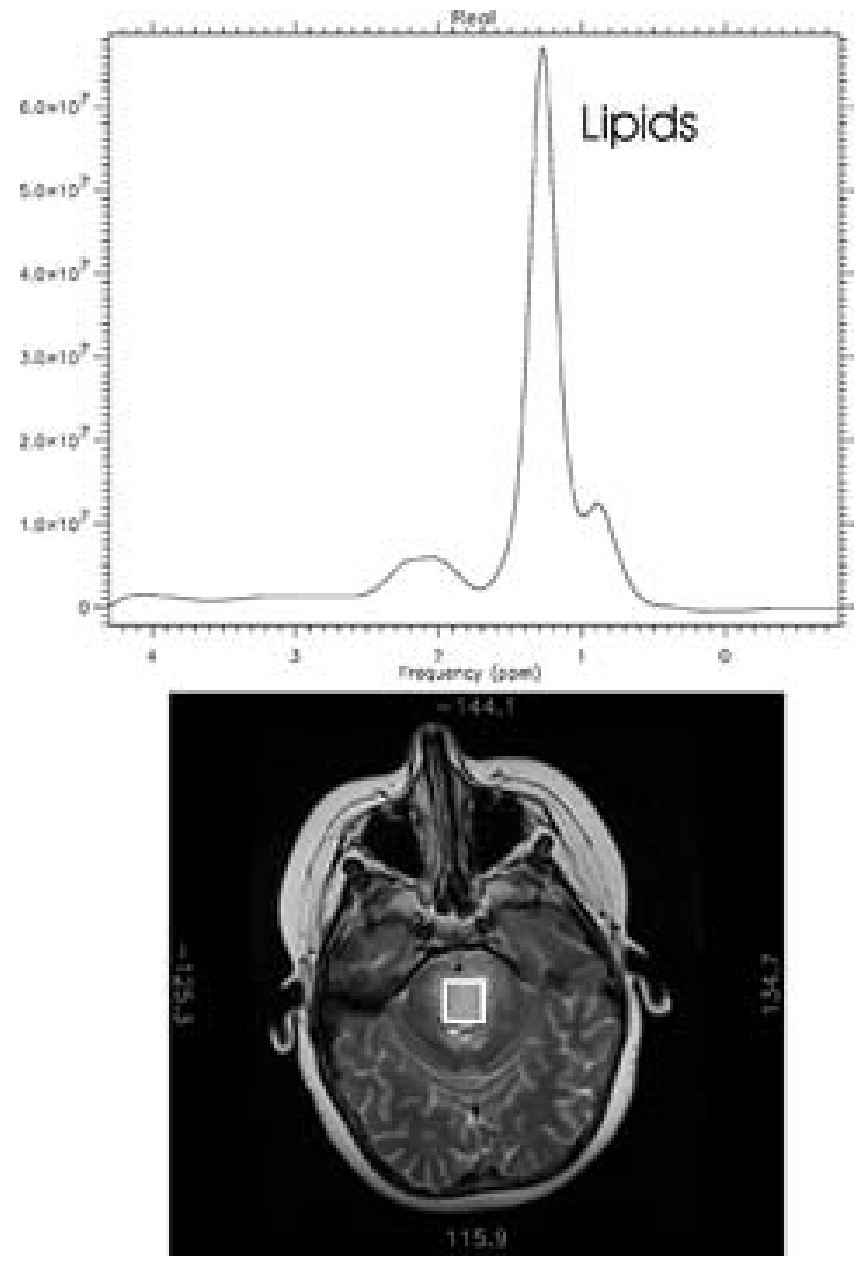

Fig. 2. Atypical pointine glioma with spectra showing the presence of lipids only.
Once selection bias is eliminated and treatment standardized, it is reasonable to assume that atypical longterm survival is based on some innate property of the tumor itself. To attempt to identify such biochemical factors, we performed MR spectroscopy to study these tumors. Magnetic resonance spectroscopy was safely and effectively performed in 17 patients with gliomas. The additional imaging sequences added fewer than 10 minutes to the overall imaging time and were obtained without sequelae.

Although these results are preliminary, they indicate that spectroscopic patterns may exist that indicate tumor behavior. In two of our patients choline, creatine, and NAA levels were low and lipid levels were elevated. Elevated choline/creatine ratios were documented in three patients when compared with normal cerebral tissue, but no significantly elevated choline/NAA ratios were found. Given the small numbers and lack of quantification of these levels, we would consider these qualitative observations. Clearly, more data are needed.

The utility of MR spectroscopy in evaluating pediatric tumors has been recognized by other authors. Warren, et al., ${ }^{24}$ demonstrated a relationship between a high choline/NAA ratio and short duration of survival in a heterogeneous group of pediatric brain tumors. They raised the issue as to whether this link may have a biological basis. They cited the fact that choline, a constituent of cell membranes, is increased in states of high membrane turnover, such as in malignant tumors. ${ }^{22}$ On the other hand, NAA is seen in normal cerebral tissue and is replaced in neoplasms that destroy such tissue. Neoplasms are thought to replace or destroy the NAA-containing cells, thereby accounting for the decreased NAA. ${ }^{16,18}$ Thus, malignant tumors with a high choline/NAA ratio would show a rapid turnover of cell membranes and decreased number of normal neurons.

Preliminary results suggesting that MR spectroscopy can yield information that differentiates among lesions in the pons may have prognostic significance.

\section{CONCLUSIONS}

Advanced neuroimaging modalities play an important 

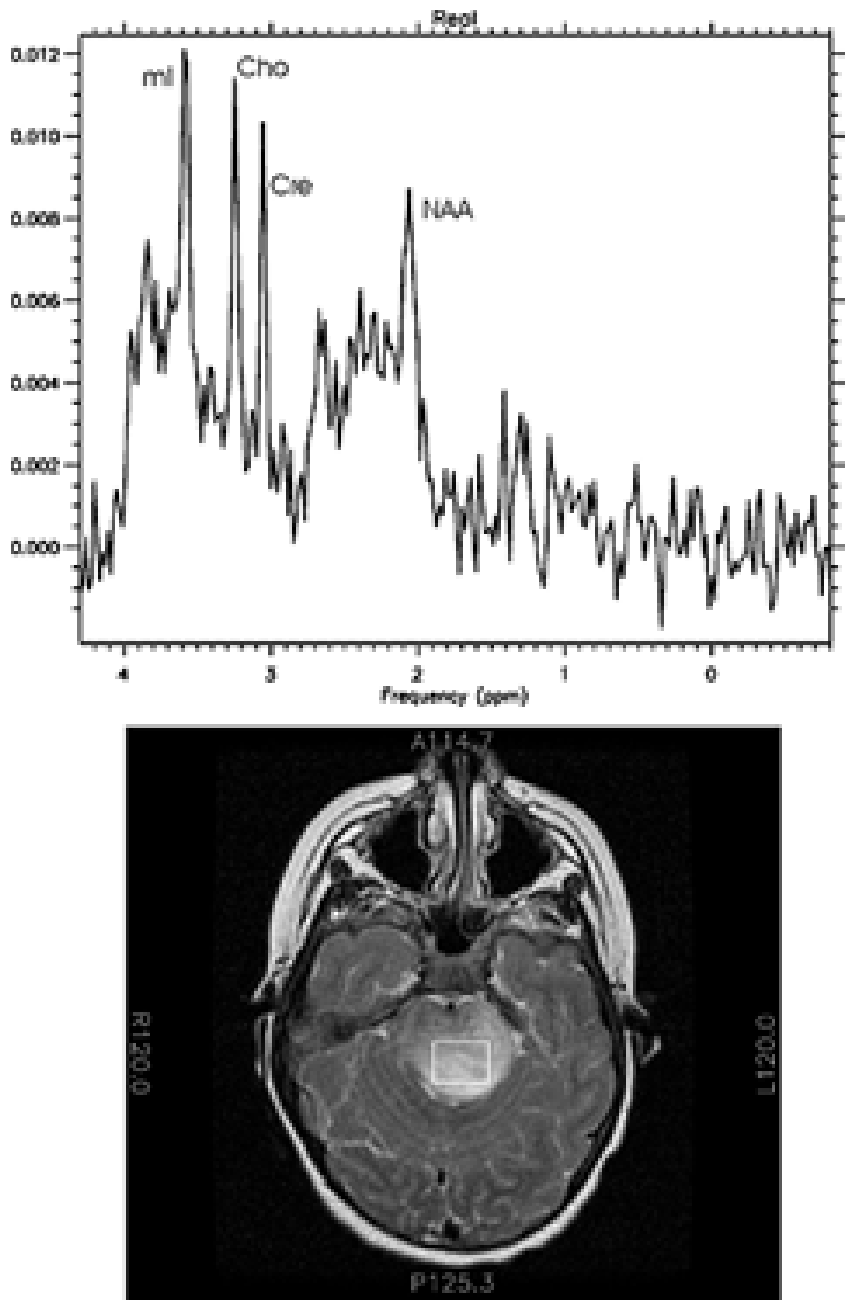

Fig. 3. Magnetic resonance spectroscopy data obtained in a patient with mildly elevated myoinositol and choline/creatine ratio.

role in the diagnosis and management of diffuse pontine gliomas. Magnetic resonance spectroscopy yields additional information that may aid in the understanding of this devastating disease.

\section{References}

1. Albright AL, Packer RJ, Zimmerman R, et al: Magnetic resonance scans should replace biopsies for the diagnosis of diffuse brain stem gliomas: a report from the Children's Cancer Group. Neurosurgery 33:1026-1030, 1993

2. Alger JR, Frank JA, Bizzi A, et al: Metabolism of human gliomas: assessment with H-1 MR spectroscopy and F-18 fluorodeoxyglucose PET. Radiology 177:633-641, 1990

3. Berger MS, Edwards MS, LaMasters D, et al: Pediatric brain stem tumors: radiographic, pathological, and clinical correlations. Neurosurgery 12:298-302, 1983

4. Bruhn H, Frahm J, Gyngell ML, et al: Noninvasive differentiation of tumors with use of localized H-1 MR spectroscopy in vivo: initial experience in patients with cerebral tumors. Radiology 172:541-548, 1989

5. Edwards MS, Wara WM, Urtasun RC, et al: Hyperfractionated radiation therapy for brain-stem glioma: a phase I-II trial. J Neurosurg 70:691-700, 1989
6. Epstein F, McCleary EL: Intrinsic brain-stem tumors of childhood: surgical indications. J Neurosurg 64:11-15, 1986

7. Ernst T, Kreis R, Ross BD: Absolute quantitation of water and metabolites in the human brain. I. Compartments and water. J Magn Reson 102:1-8, 1993

8. Farmer JP, Montes JL, Freeman CR, et al: Brainstem Gliomas. A 10-year institutional review. Pediatr Neurosurg 34: 206-214, 2001

9. Frahm J, Bruhn H, Gyngell ML, et al: Localized high-resolution proton NMR spectroscopy using stimulated echoes: initial applications to human brain in vivo. Magn Reson Med 9:79-93, 1989

10. Freeman CR, Perilongo G: Chemotherapy for brain stem gliomas. Childs Nerv Syst 15:545-553, 1999

11. Fulham MJ, Bizzi A, Dietz MJ, et al: Mapping of brain tumor metabolites with proton MR spectroscopic imaging: clinical relevance. Radiology 185:675-686, 1992

12. Hanstock CC, Rothman DL, Prichard JW, et al: Spatially localized 1H NMR spectra of metabolites in the human brain. Proc Natl Acad Sci USA 85:1821-1825, 1988

13. Kruse B, Barker PB, van Zijl PC, et al: Multislice proton magnetic resonance spectroscopic imaging in X-linked adrenoleukodystrophy. Ann Neurol 36:595-608, 1994

14. Littman P, Jarrett P, Bilaniuk LT, et al: Pediatric brain stem gliomas. Cancer 45:2787-2792, 1980

15. Marcus KJ, Dutton SC, Barnes P, et al: A phase I trial of etanidazole and hyperfractionated radiotherapy in children with diffuse brainstem glioma. Int J Radiat Oncol Biol Phys 55: 1182-1185, 2003

16. Miller BL: A review of chemical issues in 1H NMR spectroscopy: N-acetyl-L-aspartate, creatine and choline. NMR Biomed 4:47-52, 1991

17. Pierre-Kahn A, Hirsch JF, Vinchon M, et al: Surgical management of brain-stem tumors in children: results and statistical analysis of 75 cases. J Neurosurg 79:845-852, 1993

18. Ross B, Michaelis T: Clinical applications of magnetic resonance spectroscopy. Magn Reson Q 10:191-247, 1994

19. Selvapandian S, Rajshekhar V, Chandy MJ: Brainstem glioma: comparative study of clinico-radiological presentation, pathology and outcome in children and adults. Acta Neurochir 141: 721-727, 1999

20. Tate AR, Griffiths JR, Martinez-Perez I, et al: Towards a method for automated classification of 1H MRS spectra from brain tumours. NMR Biomed 11:177-191, 1998

21. Taylor JS, Ogg RJ, Langston JW: Proton MR spectroscopy of pediatric brain tumors. Neuroimaging Clin N Am 8:753-779, 1998

22. Tedeschi G, Lundbom N, Raman R, et al: Increased choline signal coinciding with malignant degeneration of cerebral gliomas: a serial proton magnetic resonance spectroscopy imaging study. J Neurosurg 87:516-524, 1997

23. Tzika AA, Vajapeyam S, Barnes PD: Multivoxel proton MR spectroscopy and hemodynamic MR imaging of childhood brain tumors: preliminary observations. AJNR 18:203-218, 1997

24. Warren KE, Frank JA, Black JL, et al: Proton magnetic resonance spectroscopic imaging in children with recurrent primary brain tumors. J Clin Oncol 18:1020-1026, 2000

25. Weaver KD, Armao D, Wiley JM, et al: Histiocytic lesion mimicking intrinsic brainstem neoplasm. Case report. J Neurosurg 91:1037-1040, 1999

Manuscript received June 11, 2003.

Accepted in final form June 20, 2003.

Address reprint requests to: Mark D. Krieger, M.D., 1300 North Vermont Avenue, Suite 1006, Los Angeles, California 90027. email: mkrieger@chla.usc.edu. 